\title{
Zufütterung von Getreide an männliche Kälber aus der Mutterkuh- haltung während der Weideperiode ${ }^{\#}$
}

\begin{abstract}
Summary
Title of the paper: Supplementation of grain to suckling beef calves during the grazing period

A study on 65 beef cow-calf pairs was conducted to investigate the intake of a feed supplementation with grain and its interrelations to performance and behaviourial characteristics of suckling calves, as well as the development of body condition and milk yield of the dam.

The intake of grain in the group receiving the supplementation (group B) was on average $2.7 \mathrm{~kg} / \mathrm{calf} / \mathrm{day}$ during the grazing period. A substantial individual variation of feed intake from $1.8 \mathrm{~kg}$ to $7.7 \mathrm{~kg}$ grain/calf/day was determined. In this period, the daily gains and the weaning weights of the calves in group B were higher (15.4\% resp. 7.4\%). The advantage of the calves of the group B could be proven only in the second half of the grazing period. The supplementation of grain caused significantly lower suckling activities and grazing periods per day. Effects of a grain supplementation in the feeding of suckling calves on pasture could not be determined on both the body condition and the milk yield of the cows.
\end{abstract}

Key Words: suckling beef calves, supplement, grazing period, beef cows

\section{Zusammenfassung}

An 65 Mutterkuh-Kalb-Paaren erfolgten Untersuchungen zur Zufutteraufnahme der Kälber und deren Wechselbeziehungen zu Leistungs- und Verhaltensmerkmalen der Kälber sowie der Körperentwicklung und Milchleistung der Mutterkühe. Während der Weideperiode nahmen die Kälber im Mittel 2,7 kg Getreide je Tier und Tag auf. Es konnten eine erhebliche tierindividuelle Variationsbreite von 1,8 kg bis zu 7,7 kg Getreide je Tier und Tag ermittelt werden. Die mit Getreide zugefütterten Kälber erreichten während der Weideperiode eine um 15,4 \% höhere tägliche Lebendmassezunahme sowie mit durchschnittlich $463 \mathrm{~kg}$ Lebendmasse ein um 7,4 \% höheres Absetzgewicht. Die Überlegenheit der Kälber mit Zufutter konnte erst in der zweiten Hälfte der Weideperiode nachgewiesen werden. Durch die Getreidezufütterung war sowohl eine signifikant geringere Säugeaktivität als auch eine signifikant verringerte Grasedauer der zugefütterten Kälber am Ende der Weideperiode erkennbar. Auswirkungen einer Zufütterung von Getreide an männliche Kälber konnten sowohl auf die Körperkondition als auch die Milchleistung der Mutterkühe nicht ermittelt werden.

Schlüsselwörter: Saugkälber, Zufütterung, Weideperiode, Mutterkühe

\section{Einleitung}

Eine hohe Leistungsausschöpfung auf der einen und eine möglichst kostensparende Produktionsorganisation auf der anderen Seite sind die Grundvoraussetzung für die wirtschaftliche Gestaltung der Mutterkuhhaltung als extensives Tierhaltungsverfahren. Einzelfütterungsversuche an Deutsch Angus- und Fleischfleckviehkühen haben bei ausschließlicher Grobfutterversorgung das rassespezifisch hohe Milchleistungsvermögen der Mutterkühe gezeigt, welches für hohe Lebendmassezunahmen der Kälber während der ersten drei Lebensmonate ausreichend ist (TEICHMANN et al., 1998). In

\footnotetext{
\# Diese Untersuchungen wurden mit Mitteln des Kultusministeriums des Landes Sachsen-Anhalt unterstützt.
} 
den ersten Lebenswochen ernähren sich gesunde Kälber ausschließlich von der Milch der Mutterkühe (BERANGER, 1990; WEIß et al., 1998). Die Milchleistung der Mutterkühe ist für das normgerechte Wachstum der Kälber in den ersten 60 bis 120 Lebenstagen ausreichend (BARTLE et al., 1984; HAMILTON und DICKIE, 1992; LOWMAN und LEWIS, 1996; ROBINSON et al., 1978; STEFLER et al., 2000; TEICHMANN, 2000). Kälber beginnen jedoch schon früher feste Futterbestandteile aufzunehmen und so decken Kälber aus der Mutterkuhhaltung im Alter von 4-5 Monaten zu ca. 50 \% ihren Energiebedarf aus der Grasaufnahme und zu ca. 50 \% über die Milch. Vom vierten bis zum neunten Lebensmonat erhöht sich der tägliche Energiebedarf von intensiv wachsenden Fleckviehbullenkälbern um 49 \% von 66 MJ ME auf 98 MJ ME (DLG, 1997). Parallel dazu sinkt die Milchleistung der Mutterkühe (GOLZE, 1999; RUPERT et al., 1999) und saisonbedingt verringert sich die Weideleistung. Daher zeigt sich in späteren Zeitabschnitten eine Abhängigkeit der täglichen Lebendmassezunahmen von der Menge und Qualität des Weidefutters (BARLETT, 2000; LE DU et al., 1981; LIPPKE et al., 2000). Am 44. Lebenstag decken die Kälber nach BAILEY und LAWSON (1981) ihren Energiebedarf zu 86 \% über die Milch ab, zum Zeitpunkt des Absetzens mit durchschnittlich 7 Monaten werden lediglich nur noch $19 \%$ der benötigten Energie über die Milch abgesichert.

In der Literatur wird eine Zufütterung der Kälber während der Weideperiode bzw. der Beginn dieser Maßnahme kontrovers diskutiert. Einige Autoren zeigen Vorteile einer frühzeitigen Zufütterung von Getreideschrot und gutem Heu an die Kälber auf, um die Entwicklung der Pansentätigkeit zu fördern (BAUER et al., 1997; HEITING, 2000; KUERT, 1999; MENKE und HUSS, 1987; WEIß et al., 1998). Auf der Weide sollen die Kälber erst dann zugefüttert werden, wenn die Milchleistung der Mutterkühe und/oder der Weideaufwuchs nicht für die Ausschöpfung des Wachstumsvermögens ausreichend erscheinen (BAUER et al., 1997; BERANGER, 1990; JEROCH et al., 2000; MARTIN und REHBOCK, 1997; MCCANN, 1995; STIEWE, 1989; WAGNER et al., 1998; WARZECHA, 1999; WEIß et al., 1998). Durch die Zufütterung wird unter extensiven Weidebedingungen eine Aufwertung der Futterration erreicht (JILG, 2001). Andere Autoren empfehlen generell eine Verabreichung von Kraftfutter im frühen Alter der Kälber (BERNER, 1975; GRAUVOGEL et al., 1997; STIEWE, 1994). Eine Zufütterung der Kälber wird weiterhin empfohlen, wenn sich die höheren Lebendmassen beim Absetzen in besseren Verkaufserlösen niederschlagen (STIEWE, 1994; WEIß et al., 1998) bzw. wirtschaftlich hohe Zunahmen bei den Kälbern erreicht werden sollen (DISSEN, 2000).

Angaben über die Höhe der aufgenommenen Zufuttermenge bei einer ad libitum Versorgung von säugenden Kälbern während der Weideperiode sind in der deutschen Literatur bisher nicht zu finden. Eine steigende tägliche Lebendmassezunahme zugefütterter Kälber von 15,6 \% bis zu 83,1 \% gegenüber den Kontrolltieren wird in der Literatur beschrieben (ADAMS, 1985; ELIZALDE et al., 1998; GALLOWAY et al., 1993; HESS et al., 1996; MYERS et al., 1999).

Die Auswirkungen einer Zufütterung an säugende Kälber auf die Entwicklung der Mutterkühe werden in der Literatur unterschiedlich beschrieben. GOLZE (1997) geht davon aus, dass zugefütterte Kälber die Milch der Mutterkühe nicht mehr in vollem Umfang nutzen und vermehrt Zufutter aufnehmen. Andere Autoren zeigen dagegen auf, dass sich die Milchaufnahme der zugefütterten Kälber nicht von der ohne Zufutter unterscheidet (EARLEY et al., 1999; LARDY et al., 2001; LUSBY, 1999). Eine Zu- 
fütterung bei Kälbern führt nach Angaben einiger Autoren (GOLZE, 1997; RASBY et al., 1991) zu steigenden Lebendmassen der Mutterkühe beim Absetzen. Unterschiede in der Körperkondition (EARLEY et al., 1999; SIMPSON et al., 1997) bzw. der Lebendmasse der Mutterkühe (LUSBY, 1999; STROH et al., 2001) beim Absetzen in Abhängigkeit einer Zufütterung der Kälber konnten genannte Autoren nicht beobachten.

Das Ziel der vorliegenden Untersuchung war es, folgende Fragestellungen zu bearbeiten:

1. Untersuchungen zum Einfluss der Getreidezufütterung auf die Leistung und das Verhalten von männlichen Kälbern aus der Mutterkuhhaltung bis zum Ende der Weideperiode,

2. Charakterisieren der Wechselbeziehungen zwischen Mutterkuh und Kalb in Abhängigkeit des Weidehaltungsverfahrens der Kälber,

3. Ermittlung der Wechselbeziehungen zwischen den Leistungsmerkmalen in der Weide- und der anschließenden Intensivmastperiode.

Im Mittelpunkt der Untersuchung stand die für das Verfahren der Mutterkuhhaltung und anschließende Intensivmast wirtschaftlich bedeutungsvolle Frage nach der Notwendigkeit des Ausgleiches von Energiedefiziten bei Kälbern auf Spätherbstweiden, um hohe Schlachtkörperqualitäten zu erzielen und die Körperkonditionsentwicklung der Mutterkühe verfahrensgerecht zu gestalten.

\section{Material und Methode}

In die Untersuchungen wurden aus den Kalbejahren 1999 und 2000 insgesamt 65 Mutterkuh-Kalb-Paare einbezogen. Die männlichen Kälber aus der Anpaarung mit Charolais wurden im Zeitraum von Januar bis März geboren. Zum Zeitpunkt des Versuchsbeginns mussten alle Mutterkühe wieder tragend sein. Die Weidebedingungen werden durch Mineralbödengrünland der altmärkischen Wische mit guten Weidegrasbeständen charakterisiert. Im Mittel beider Versuchsjahre konnten 79 dt Trockenmasse bzw. 48.000 MJ NEL je Hektar ermittelt werden. Mit einem mittleren Energiegehalt von $10 \mathrm{MJ}$ ME stand bei schwankenden Mengenerträgen auf ca. 1,4 ha je MutterkuhKalb-Paar eine gute Weidefutterqualität zur Verfügung. In einer Vorperiode bis zum durchschnittlich 131. Säugetag erfolgte die Haltung aller Tiere unter einheitlichen Bedingungen. Danach wurden zwei Gruppen mit gleicher Anzahl Mutterkuh-Kalb-Paare gebildet. Die Kälber der Gruppe A ernährten sich ausschließlich vom Weidefutter und der Milch der Mutterkühe. Den Kälbern der Gruppe B wurde zusätzlich die Aufnahme von Zufutter ad libitum in Form von gequetschter Gerste ermöglicht (Tab. 1).

Tabelle 1

Versuchsdesign (Design of investigation)

\begin{tabular}{|c|c|c|c|c|c|c|c|c|c|c|c|c|}
\hline Kühe / Kälber & \multicolumn{12}{|c|}{65 / 65} \\
\hline Versuchsjahr & \multicolumn{6}{|c|}{$1999(n=28)$} & \multicolumn{6}{|c|}{$2000(n=37)$} \\
\hline Weidehaltung & \multicolumn{3}{|c|}{$\begin{array}{l}\text { ohne Zufutter } \\
\text { Gruppe (A) } \\
(n=14)\end{array}$} & \multicolumn{3}{|c|}{$\begin{array}{c}\text { mit Zufutter } \\
\text { Gruppe (B) } \\
(\mathrm{n}=14)\end{array}$} & \multicolumn{3}{|c|}{$\begin{array}{l}\text { Ohne Zufutter } \\
\text { Gruppe (A) } \\
(n=19)\end{array}$} & \multicolumn{3}{|c|}{$\begin{array}{c}\text { mit Zufutter } \\
\text { Gruppe (B) } \\
(\mathrm{n}=18)\end{array}$} \\
\hline Kalbenummer & $1-3$ & $4+5$ & $\geq 6$ & $1-3$ & $4+5$ & $\geq 6$ & $1-3$ & $4+5$ & $\geq 6$ & $1-3$ & $4+5$ & $\geq 6$ \\
\hline
\end{tabular}

Im Versuchsjahr 1999 kam ein handelsüblicher, doppelseitiger Futterautomat zum Einsatz. Die mittlere Zufutteraufnahme je Kalb und Tag wurde durch die tägliche Einund Auswaage des Zufutters in den späten Abendstunden als Gruppenmittelwert er- 
fasst. Um eine tierindividuelle Erfassung des Zufutterverzehrs zu ermöglichen, fand im zweiten Versuchsjahr eine für die Versuchsanstellung entwickelte Fütterungseinrichtung mit 8 Einzelfutterboxen Verwendung. Beim Betreten der Box wird das Kalb identifiziert und diesem nach vorgegebenen Parametern Futter zugeteilt. Die vom Kalb aufgenommenen Mengen wurden mittels einer speziellen Software gespeichert und ausgewertet.

Das Säugeverhalten der Kälber wurde während der Lichtphase des Tages nach der Ereignis-Teil-Methode (FASSNACHT, 1979) mindestens im zweiwöchentlichen Intervall erfasst. Im Oktober 2000 konnte das Graseverhalten an insgesamt 8 ausgewählten Kälbern beider Gruppen mittels der Zeit-Teil-Methode ermittelt werden. Über die gesamte Lichtphase des Tages erfolgte die Dokumentation ausgewählter Verhaltensweisen der Kälber (Grasen, Säugen, Zufutteraufnahme sowie sonstige Verhaltensweisen) im 5-Minuten-Intervall. Die Milchleistung der Mutterkühe wurde im vierwöchentlichen Intervall ab der 4. Woche post partum im Versuchsjahr 2000 mittels maschinellem Milchentzug unter Anwendung von 20 IE Oxytocin erfasst (Tab. 2).

Tabelle 2

Ausgewählte Untersuchungsmerkmale, deren Frequenz und Methoden (Selected characteristics of investigation, frequency and methods)

\begin{tabular}{|c|c|c|c|}
\hline & Merkmal & Frequenz & Methode \\
\hline \multirow{5}{*}{ 离 } & Lebendmasse & vierwöchentlich & Wägung \\
\hline & Zufutteraufnahme & 1999 - täglich & Ein- und Rückwaage (Gruppenmittelwert) \\
\hline & & 2000 - täglich & PC-Erfassung (tierindividuell) \\
\hline & Graseverhalten & 4x; Oktober 2000 & Zeit-Teil-Methode durch Direktbeobachtung (5- \\
\hline & Säugeverhalten & zweiwöchentlich & $\begin{array}{l}\text { Ereignis-Teil-Methode durch Direktbeobachtung; } 13 \\
\text { bis } 20 \text { Stunden je Tag }\end{array}$ \\
\hline \multirow{4}{*}{ 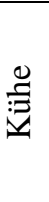 } & Lebendmasse (LM) & vierwöchentlich & Wägung \\
\hline & Rückenfettdicke (RFD) & vierwöchentlich & Ultraschallmessung; nach STAUFENBIEL (1992) \\
\hline & Milchmenge & vierwöchentlich & $\begin{array}{l}\text { Maschineller Milchentzug mit Einsatz von } 20 \text { IE } \\
\text { Oxytocin; nach BEAL et al. (1990) }\end{array}$ \\
\hline & Milchinhaltsstoffe & vierwöchentlich & Infrarotanalyse \\
\hline
\end{tabular}

Die Jungbullen wurden von ihren Müttern bei Weideabtrieb Ende Oktober bzw. Anfang November mit einem mittleren Alter von 268 Tagen getrennt. Danach wurden sie in einer 180-Tage-Intensivmast analog ihrer Vorbehandlung während der Weideperiode auf Mast- und Schlachtleistung unter einheitlichen Versuchsbedingungen geprüft. Die Erfassung sowie Aufbereitung der Daten erfolgte mit dem Tabellenkalkulationsprogramm EXCEL 7.0 (Microsoft ${ }^{\circledR}$ Corporation, 1997). Für die statistische Auswertung wurden die Programme STATISTICA 5.1 (StatSoft $^{\circledR}$, 1996) sowie SAS (SAS Institute Inc., 1999) genutzt. Die Korrelationskoeffizienten wurden nach den Methoden von Pearson und Spearman berechnet. Die Gruppen wurden auf signifikante Mittelwertdifferenzen mit einer Irrtumswahrscheinlichkeit von $\mathrm{p} \leq 0,05$ getestet. In den Tabellen erscheinen die Signifikanzsymbole als Exponenten.

\section{Ergebnisse und Diskussion}

Im Mittel der Weideperiode nahmen die Kälber 2,7 kg Zufutter je Tier und Tag auf. Der Verlauf der mittleren täglichen Zufutteraufnahme war gekennzeichnet durch einen stetigen Anstieg über die einzelnen Monate der Weideperiode. Die Summe der tierindividuellen Zufutteraufnahme je Kalb variierte im Versuchsjahr 2000 von 165 kg bis 
zu 696 kg, was einer mittleren täglichen Aufnahme von 1,8 kg bzw. 7,7 kg entspricht. Eine mittlere Besuchshäufigkeit der Kälber am Zufutterautomaten von 2,6 \pm 1,8 Besuchen je Tier und Tag konnte im Versuchsjahr 1999 ermittelt werden (Tab. 3). Im Oktober 2000 wurden mit $\bar{x}=3,3 \pm 1,6$ Automatenbesuchen je Tier und Tag vergleichbare Ergebnisse gegenüber denen im Oktober 1999 dokumentiert.

Tabelle 3

Zufutteraufnahme der Kälber und Automatenbesuche (Intake of supplement through the beef calves and stays to the feeding station)

\begin{tabular}{|c|c|c|c|c|c|c|}
\hline & \multicolumn{4}{|c|}{1999} & \multirow{2}{*}{\multicolumn{2}{|c|}{$\begin{array}{c}2000 \\
\text { kg / Kalb / Tag }\end{array}$}} \\
\hline & \multicolumn{2}{|c|}{ kg / Kalb / Tag } & \multicolumn{2}{|c|}{ Besuchshäufigkeit } & & \\
\hline & $\bar{x}$ & $\mathrm{~s}^{1}$ & $\bar{x}$ & $\mathrm{~s}$ & $\bar{x}$ & $\mathrm{~s}$ \\
\hline Juni & & & & & 0,39 & $0,31^{1}$ \\
\hline Juli & 0,52 & 0,23 & 0,5 & 1,2 & 1,13 & $0,89^{1}$ \\
\hline August & 1,46 & 1,20 & 1,8 & 1,2 & 2,44 & $2,42^{2}$ \\
\hline September & 2,84 & 1,02 & 2,4 & 1,4 & 4,59 & $3,74^{2}$ \\
\hline Oktober & 5,76 & 0,98 & 3,6 & 1,8 & 6,05 & $3,93^{2}$ \\
\hline
\end{tabular}

${ }^{1}$ Standardabweichung der Tageswerte; ${ }^{2}$ Standardabweichung der Einzeltiere

Gesicherte Beziehungen der tierindividuell erfassten Zufuttermenge bestanden zur 100-Tage-Milchleistung der Mutterkühe $\left(\mathrm{r}=0,479^{*}\right)$ und zur täglichen Lebendmassezunahme der Kälber in der Vorperiode $\left(\mathrm{r}=0,493^{*}\right)$. Zwischen mittlerer täglicher Lebendmassezunahme der Kälber während der Weideperiode und der Zufuttermenge konnte eine Korrelationskoeffizient von r=0,356 ermittelt werden. Auch in der Literatur wird eine positive Beziehung zwischen aufgenommener Zufuttermenge und der Lebendmassezunahme der Kälber beschrieben (LUSBY et al., 1976; WYATT et al., 1977). Dagegen war in den eigenen Untersuchungen der Zusammenhang zwischen Milchleistung der Mutterkühe und Zufutteraufnahme der Kälber von r=0,220 geringer. Andere Autoren weisen negative Beziehungen aus (FISS und WILTON, 1992; HAMILTON und DICKIE, 1988; LUSBY et al., 1976; MCMORRIS und WILTON, 1986). Mit steigender Milchleistung der Kühe können die Kälber höhere Futtermengen aufnehmen (CHRISTIAN et al., 1965; FISS und WILTON, 1992), da diese über eine höhere Wachstumskapazität verfügen. Aus den beschriebenen Zusammenhängen lässt sich schlussfolgern, dass das Leistungspotential der Kälber die Aufnahme an Zufutter am stärksten beeinflusst.

In der einheitlichen Vorperiode betrug die mittlere tägliche Lebendmassezunahme aller Kälber $\bar{x}=1371 \mathrm{~g} \pm 188 \mathrm{~g}$. Während der Weideperiode lagen die mittleren täglichen Lebendmassezunahmen der zugefütterten Kälber mit $1728 \mathrm{~g}$ um 15,4\% über der Leistung der Vergleichstiere ohne Zufütterung. Beim Absetzen erreichten die zugefütterten Kälber 7,4 \% höhere Absetzgewichte und eine leicht bessere Körperkondition als die Tiere der Kontrollgruppe (Tab. 4).

Tabelle 4

Leistungsparameter der Kälber während der Weideperiode ( $n=65)$ (Parameters of beef calves during the grazing season)

\begin{tabular}{lrrrr}
\hline & Gruppe A & \multicolumn{2}{c}{ Gruppe B } \\
\hline Alter zu Versuchsbeginn (d) & $131 \pm 25$ & 19,1 & $132 \pm 24$ & 18,2 \\
LM zu Versuchsbeginn (kg) & $225 \pm 49$ & 21,8 & $225 \pm 41$ & 18,2 \\
LM beim Absetzen (kg) & $431^{\mathrm{a}} \pm 61$ & 14,2 & $463^{\mathrm{b}} \pm 55$ & 11,9 \\
Lebendmassezunahme (g) & $1498^{\mathrm{a}} \pm 160$ & 10,7 & $1728^{\mathrm{b}} \pm 171$ & 9,9 \\
RFD beim Absetzen (mm) & $9,9 \pm 1,6$ & 16,2 & $10,5 \pm 1,7$ & 16,2 \\
\hline
\end{tabular}


Signifikante Unterschiede in den mittleren täglichen Lebendmassezunahmen der Kälber konnten erst in der zweiten Hälfte der Weideperiode nachgewiesen werden. Hier erreichten die zugefütterten Kälber eine um 38,9 \% höhere Leistung gegenüber den Vergleichstieren ohne Zufütterung (Tab. 5).

Tabelle 5

Tägliche Lebendmassezunahme der Kälber bei Gruppierung der Weideperiode in zwei Hälften (n=65) (Daily live weight gain of beef calves by sorting the grazing season in two parts)

\begin{tabular}{lrrrr}
\hline & \multicolumn{2}{c}{ Gruppe A } & \multicolumn{2}{c}{ Gruppe B } \\
\hline 1. Hälfte Weideperiode & $1693 \mathrm{~g} \pm 170 \mathrm{~g}$ & $10,0 \%$ & $1698 \mathrm{~g} \pm 231 \mathrm{~g}$ & $13,6 \%$ \\
2. Hälfte Weideperiode & $1293^{\mathrm{a}} \mathrm{g} \pm 249 \mathrm{~g}$ & $19,3 \%$ & $1796^{\mathrm{b}} \mathrm{g} \pm 238 \mathrm{~g}$ & $13,3 \%$ \\
\hline
\end{tabular}

Die zweite Hälfte der Weideperiode ist durch eine um 5,8 \% höhere tägliche Lebendmassezunahme der zugefütterten Kälber gegenüber der 1 . Hälfte charakterisiert. In der Gruppe A verringerten sich die täglichen Lebendmassezunahmen auf 76,4\% in der zweiten Hälfte der Weideperiode gegenüber der in den vorangegangenen Abschnitten erbrachten Leistung.

Die Zufütterung der Kälber während der Weideperiode führte zu einer Veränderung des Säugeverhaltens. Signifikant niedrigere Säugefrequenzen der zugefütterten Kälber ließen sich zum Ende der Weideperiode in den Monaten September und Oktober gegenüber den Kälbern ohne Zufütterung beobachten (Abb. 1).

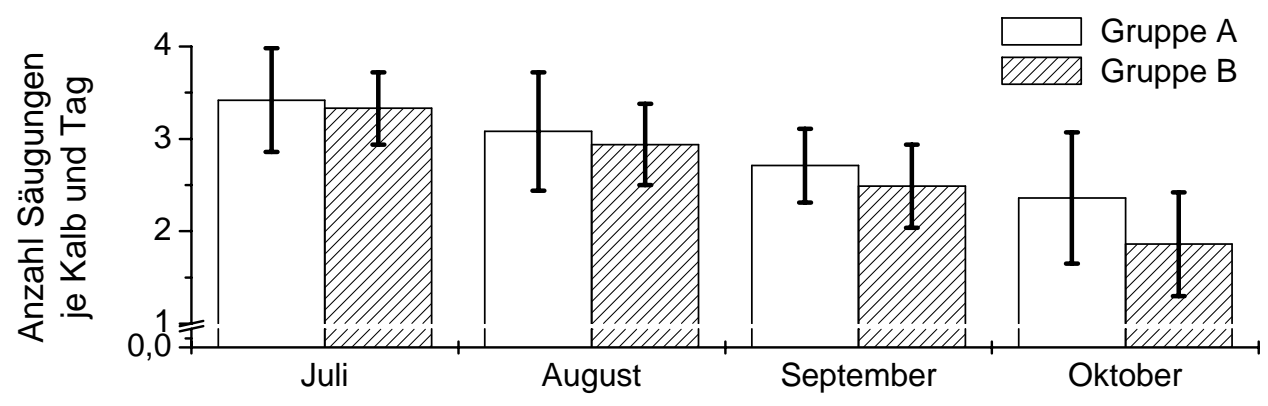

Abb. 1: Mittlere Anzahl Säugungen je Kalb und Tag während der Weideperiode (Average numbers of suckling each calf and day)

Eine Verringerung der Säugeaktivitäten durch eine Zufütterung von mineralisiertem Kraftfutter wurde auch von MÄNNER und LAIBLIN (2000) beschrieben. Ursächlich für die signifikant geringere Säugefrequenz in den Untersuchungen könnte eine verbesserte energetische Versorgung der zugefütterten Kälber sein, die die abnehmende Milchleistung der Mutterkühe, die Verringerung des Weidefutterangebotes sowie Veränderungen der Tagesrhythmik durch die Aufnahme an Zufutter ausgleichen können.

Die zugefütterten Kälber grasten im Oktober 2000 mit einer um 217 min signifikant verringerten Zeitdauer gegenüber den Vergleichstieren ohne Zufütterung (Tab. 6).

Auch andere Autoren geben eine verringerte Grasezeit zugefütterter Kälber an (BODINE et al., 2000; HESS et al., 1994; KRYSL und HESS, 1993; WAGNER et al., 1998). Neben der Zufütterung zeigen jedoch weitere Einflussgrößen wie das angebotene Weidefutter (SCARNECCHIA et al., 1985), die Häufigkeit der Zufutteraufnahme (BEATY et al., 1994) sowie das Weidemanagement (KRYSL und HESS, 1993) Auswirkungen auf die Ergebnisse der Beobachtungen. 
Tabelle 6

Grasezeiten der Kälber im Oktober (n=8) (Grazing time of beef calves in October)

\begin{tabular}{lcccc}
\hline & \multicolumn{3}{c}{ Gruppe A } & \multicolumn{2}{c}{ Gruppe B } \\
\hline Grasezeit absolut (min/d) & $370^{\mathrm{a}} \pm 59$ & 16,0 & $153^{\mathrm{b}} \pm 81$ & 52,9 \\
Grasezeit relativ (\%) $*^{1}$ & $47,4^{\mathrm{a}} \pm 5,8$ & 12,2 & $19,7^{\mathrm{b}} \pm 9,2$ & 46,7 \\
\hline${ }^{* 1}$ relativer Anteil an der Lichtphase des Tages & & & &
\end{tabular}

Die Mutterkühe der Versuchsgruppe erreichten bei geringerer Lebendmasse zu Versuchsbeginn gegenüber den Mutterkühen der Kontrollgruppe eine vergleichbare Lebendmasse beim Absetzen der Kälber. Signifikante Unterschiede bei den Merkmalen der Mutterkühe ließen sich zwischen beiden Gruppen während der Weideperiode nicht nachweisen. Die Mutterkühe beider Gruppen wiesen einen Aufbau an Lebendmasse bei gleichzeitiger Mobilisierung von Rückenfett während der Weideperiode auf (Tab. 7).

Tabelle 7

Lebendmasse und Rückenfettdicke der Mutterkühe während der Weideperiode (n=65) (Live weight and backfat thickness of beef cows during the grazing season)

\begin{tabular}{lcrrr}
\hline & \multicolumn{1}{c}{ Gruppe A } & \multicolumn{2}{c}{ Gruppe B } \\
\hline Lebendmasse Versuchsbeginn (kg) & $684 \pm 67$ & 9,8 & $678 \pm 62$ & 9,1 \\
Lebendmasse Absetzen (kg) & $701 \pm 67$ & 9,6 & $700 \pm 57$ & 8,1 \\
Rückenfettdicke Versuchsbeginn & $19,8 \pm 7,4$ & 37,4 & $19,1 \pm 6,6$ & 34,6 \\
(mm) & & & & \\
Rückenfettdicke Absetzen (mm) & $16,0 \pm 6,3$ & 39,4 & $17,0 \pm 6,9$ & 40,6 \\
\hline
\end{tabular}

Die in der Literatur beschriebenen Zusammenhänge zwischen einer Zufütterung der Kälber und höheren Lebendmassen der Mutterkühe beim Absetzen (FAULKNER et al., 1994; GOLZE, 1997; RASBY et al., 1991) bzw. einer höheren Körperkondition der Kühe zu diesem Zeitpunkt (CARLYON, 1998; MARTIN, 2000; PRICHARD et al., 1989) konnten auf der Grundlage des eigenen Zahlenmaterials nicht beobachtet werden. Dies bestätigt die Angaben anderer Autoren (EARLEY et al., 1999; LUSBY, 1999; SIMPSON et al., 1997; TARR et al., 1994; TJARDES et al., 1998).

Im Zeitraum der 155-tägigen Weideperiode des Versuchsjahres 2000 konnte eine mittlere tägliche Milchmenge der Mutterkühe der Kontrollgruppe A von 11,4 kg ermittelt werden. Die Mutterkühe der Versuchsgruppe B erreichten mit 12,2 kg Milch je Tag eine etwas höhere Milchleistung (Abb. 2).

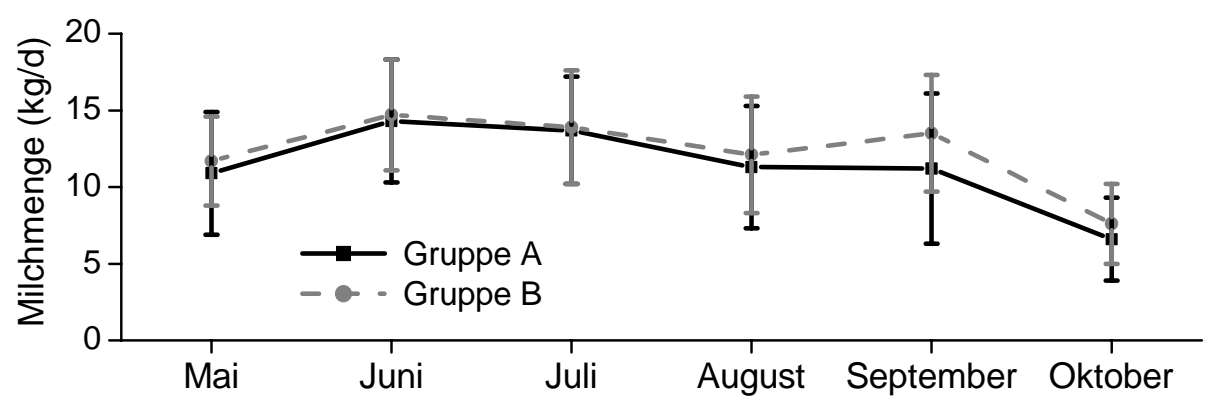

Abb. 2: Milchleistung der Mutterkühe während der Weideperiode des Versuchsjahres 2000 ( $n=37)$ (Milk yield of beef cows during the grazing season) 
Aus Abbildung 2 kann geschlussfolgert werden, dass die zugefütterten Kälber die Milch der Mutterkühe in vollem Umfang nutzen, was die Literaturangaben anderer Autoren bestätigt (EARLEY et al., 1999; LARDY et al., 2001; LUSBY, 1999).

\section{Schlussfolgerungen}

Aus den vorliegenden Ergebnissen lassen sich folgende Schlussfolgerungen ableiten:

1. Säugeperioden von 8 bis 9 Monaten Dauer bei 5 bis 6 Monaten Weidegang mit ständig ausreichendem Weidefutterangebot sind wesentliche Voraussetzungen für eine futterkosteneffiziente, leistungsorientierte Gestaltung der Mutterkuhhaltung mit nachfolgender Intensivmast im eigenen Unternehmen.

2. Auf Spätherbstweiden nehmen männliche Mutterkuhkälber neben der Muttermilch nicht genügend Weidegras zur Ausschöpfung ihres Wachstumsvermögens auf. Für den Energie- und Nährstoffausgleich stellt die Zufütterung von Getreide eine Alternative dar.

3. Die Grasedauer und die Weidefutteraufnahme der Kälber werden durch die ad libitum Zufütterung von Getreide wesentlich verringert. Die höheren Weidereste können durch eine höhere Besatzstärke oder besser eine Verlängerung der Weideperiode für die Mutterkühe nach dem Absetzen genutzt werden.

4. Die Getreidezufütterung an Kälber während der Weideperiode hat keinen Einfluss auf die Körperkonditionsentwicklung der Mutterkühe.

5. Mutterkühe der intensiven Fleischrindrassen verfügen über ein hohes Milchleistungsvermögen bei guten Persistenzen. Soll das Milchleistungspotential der Mutterkühe mit Januar- bzw. Februarabkalbung durch die Kälber genutzt werden, dürfen diese erst im Oktober von ihren Müttern getrennt werden.

\section{Literatur}

ADAMS, D.C.:

Effect of time of supplementation on performance, forage intake and behavior of yearling beef steers grazing russian wild ryegrass in the fall; J. Anim. Sci. 61 (1985), 1037-1042

BAILEY, C.B.; LAWSON, J.E.:

Estimated water and forage intake in nursing beef calves; Can. J. Anim. Sci. 61 (1981), 415-424

BARTLE, S.J.; MALES, J.R.; PRESTON, L.R.:

Effect of energy intake on the post partum interval in beef cows and the adequacy of the cows milk production for calf growth; J. Anim. Sci. 58 (1984), 1068-1074

BARTLETT, B.:

Drought - Cattle and grass management; Department of Animal Science, Michigan State Univ., East Lancing (2001)

BAUER, K.; STEINWEDER, R.; STODULKA, R.:

Mutterkuhhaltung; Leopold Stocker Verlag, Graz - Stuttgart (1997)

BEATY, J.L.; COCHRAN, R.C.; LINTZENICH, B. A.; VANZANT, E.S.; MORRILL, J.L.; BRANDT, R.T.; JOHNSON, D.E.:

Effect of frequency of supplementation and protein concentration in supplements on performance and digestion characteristics on beef cattle consuming low-quality forages; J. Anim. Sci. 72 (1994), 24752486

BERANGER, C.:

Kälber säugender Kühe; In MORNET, P.; ESPINASE, J.: Das Kalb; Schober Verlags GmbH (1990)

BERNER, D.:

Kraftfutterzufütterung bei der Bullenmast auf der Weide?; Der Tierzüchter 27 (1975), 107-110

BOCK, B.J.; HANNAH, S.M.; BRAZLE, F.K.; CORAH, L.R.; KUHL, G.L.:

When are protein and energy supplements needed?; in: Stocker Cattle Management \& Nutrition, Kansas State Univ. (1991)

BODINE, T.N.; PURVIS, H.T.; COX, D.A.: 
Effects of supplemental energy and degradable intake protein on grazing behavior, forage intake, digestion and performance of steers grazing winter range; Oklahoma State Univ., Animal Science Research Report (2000), 33-39

CARLYON, R.:

Creep feeding calves; Alberta Agriculture, Food and Rural Development, Westlock (1998)

DISSEN, J.:

Die Erfolgreichen schwören auf hohe Absetzerqualität; top agrar 2 (2000), 26-29

DLG:

Leistungs- und Qualitätsgerechte Bullenmast - Vorgaben zur Anwendung der Umsetzbaren Energie (ME); Deutsche Landwirtschaftsgesellschaft (1997)

EARLEY, A. V.; SOWELL, B. F.; BOWMAN, J. G. P.:

Liquid supplementation of grazing cows and calves; Animal feed science and technology 80 (1999) 3-4, 281-296

ELIZALDE, J.C.; CREMIN, J.D.; FAULKNER, D.B.; MERCHEN, N.R.:

Performance and digestion by steers grazing tall fescue and supplemented with energy and protein; J. Anim. Sci. 76 (1998), 1691-1701

FASSNACHT, G.:

Systematische Verhaltensbeobachtung; Reinhardt-Verlag, München und Basel (1979)

FAULKNER, D.B.; HUMMEL, D.F.; BUSKIRK, D.D.; BERGER, L.L.; PARRETT, D.F.; CMARIK, G. F.: Performance and nutrient metabolism by nursing calves supplemented with limited or unlimited corn or soybulls; J. Anim. Sci. 72 (1994), 470-477

FISS, C.F.; WILTON, J.W.:

Contribution of breed, cow weight, and milk yield to the traits of heifers and cows in four beef breeding systems; J. Anim. Sci. 70 (1992), 3686-3696

GALLOWAY, D.L.; GOETSCH, A.L.; FORSTER, L.A.; BRAKE, A.C.; JOHNSON, Z.B.:

Digestion, feed intake, and live weight gain by cattle consuming bermudagrass and supplemented with different grains; J. Anim. Sci. 71 (1993) 5, 1288-1297

GOLZE, M.:

Extensive Rinderhaltung; Fleischrinder - Mutterkühe; Rassen, Herdenmanagement, Wirtschaftlichkeit; Verlags Union Agrar (1997)

GOLZE, M.: Kälbern in der Säugeperiode bei Fleischrindern zufüttern?; REKASAN-Journal 6 (1999), 114-118

GRAUVOGL, A.; PIRKELMANN, H.; ROSENBERGER, G.; V. ZERBONI DI SPOSETTI, H.-N.: Artgemäße und rentable Nutztierhaltung; Verlags Union Agrar (1997)

HAMILTON, T.; DICKIE, D.:

HEITING, N.: Creep feeding beef calves; Ministry of Agriculture, Food and Rural Affairs of Ontario (1988)

Kälbergesundheit - Haltungs- und Fütterungskonzepte für Kälber; Züchtungskunde 72 (2000), 450-458

HESS, B.W.; PARK, K.K.; KRYSL, L.J.; JUDKINS, M.B.; MCCRACKEN, B.A.; HANKS, D.R.:

Supplemental protein for beef cattle grazing dormant intermediate wheatgrass pasture: effects on nutrient quality, forage intake, digesta kinetics, grazing behavior, ruminal fermentation, and digestion; J. Anim. Sci. 72 (1994) 8, 2113-2123

HESS, B.W.; KRYSL, L.J.; JUDKINS, M.B.; HOLCOMBE, D.W.; HESS, J.D.; HANKS, D.R.; HUBER, S.A.: Supplemental cracked corn or wheat bran for steers grazing endophyte-free fescue pasture: effects on live weight gain, nutrient quality, forage intake, particulate and fluid kinetics, ruminal fermentation, and digestion; J. Anim. Sci. 74 (1996) 5, 1116-1125

JEROCH, H.; DROCHNER, W.; SIMON, O.:

JILG, T.: Ernährung landwirtschaftlicher Nutztiere; UTB-Verlag (2000)

Auswahl geeigneter Rinderrassen für Beweidungsprojekte; Naturschutz-Info 1 (2001)

KRYSL, L.J.; HESS, B.W.: KUERT, J.: Influence of supplementation on behavior of grazing cattle; J. Anim. Sci. 71 (1993) 9, 2546-2555

Das erste Kalb mit 24 Monaten; UFA - Revue 03 (1999), 2-3

LARDY, G.P.; ADAMS, D.C.; KLOPFENSTEIN, T.J.; CLARK, R.T.; EMERSON, J.: Escape protein and weaning effects on calves grazing meadow regrowth; J. Range Manage. 54 (2001), 233-238

LE DU, Y.L.; BAKER, R.D.; NEWBERRY, R.D.:

Herbage intake and milk production by grazing dairy cows, 3 . The effect of grazing severity under continuous stocking; Grass Forage Sci. 36 (1981), 307-318

LIPPKE, H.; FORBES, T.D.A.; ELLIS, W.C.: 
Effect of supplements on growth and forage intake by stocker steers grazing wheat pasture; J. Anim. Sci. 78 (2000) 6, 1625-1635

LOWMAN, B.G.; LEWIS, M.: Feeding the beef suckler herd; XIX World Buiatrics Congress, Edinburgh (1996)

LUSBY, K.S.; STEPHEN, D.F.; TOTUSEK, R.:

Effects of milk intake by nursing calves on forage intake on range and creep intake and digestibility in dry lot; J. Anim. Sci. 43 (1976), 1066-1071

LUSBY, K. S.:

Creep feeding beef calves; Oklahoma State Univ., Circular E-848 (1999)

MÄNNER, K.; LAIBLIN, C.:

Mineral- und Spurenelementversorgung von Mutterkühen und Kälbern; 9. Sächsischer Fleischrindtag, Raschau, 30. September 2000

MARTIN, J.; REHBOCK, F.:

Zuchtleistung von Mutterkühen unter Berücksichtigung des Erstkalbealters; Arch. Tierz., Dummerstorf

MARTIN, J.: 40 (1997), Sonderheft, 201

Fütterung von Fleischrindzuchtbullen in der Eigenleistungsprüfung; Infoheft FRV 1 (2000)

MCCANN, M.A.:

Creep feeding beef calves; Univ. of Georgia - College of Agricultural \& Environmental Science Georgia Extension Service publications (1995)

MCMORRIS, M.R.; WILTON, J.W.:

Breeding system, cow weight and milk yield effects on various biological variables in beef production; J. Anim. Sci. 63 (1986), 1361-1372

MENKE, K.-H.; HUSS, W.:

Tierernährung und Futtermittelkunde; Verlag Eugen Ulmer, Stuttgart (1987)

MYERS, S.E.; FAULKNER, D.B.; NASH, T.G.; BERGER, L.L.; PARRETT, D.F.; MCKEITH, F.K.:

Performance and carcass traits of early-weaned steers receiving either a pasture growing period or a finishing diet at weaning; J. Anim. Sci. 77 (1999), 311-322

PRICHARD, D.L.; HARGROVE, D.D.; OLSON, T.A.; MARSHALL, T.T.:

Effects of creep feeding, zeranol implants and breed type on beef cattle production; I. Calf and cow performance; J. Anim. Sci. 67 (1989), 609-616

RASBY, R.; GOSEY, J.; RUSH, I.:

Creep feeding beef calves; University of Nebraska, Lincoln (1991)

ROBINSON, O.W.; YUSUFF, M.K.M.; DILLARD, E.U.:

Milk production in Hereford cows I. means and correlations; J. Anim. Sci. 47 (1978), 131-136

RUPERT, S.D.; BUCHANAN, D.S.; MINICK, J.A.; KNORI, L.:

Use of milk EPDs to predict differences in milk production of range beef cows. Oklahoma State Univ., Animal Science Research Report (1999), 9-12

SCARNECCHIA, D.L.; NASTIS, A.S.; MALECHEK, J.C.:

Effects of forage availability on grazing behavior of heifers; J. Range Manage. 38 (1985), 177-180

SIMPSON, R.B.; PHILLIPS, J.M.; ROSENKRANS, C.F.; BROWN, A.H.; KOLLEGG, D.W.:

Development of replacement heifers using combinations of three forage types and feed supplements (with or without broiler litter); Arkansas Experiment Station Bulletin 955 (1997)

STEFLER, J.; KOVÁCS, A. Z.; FAHR, R.-D.; SCHOLZ, H.; TEICHMANN, S.:

Milchleistung und Milchqualität von Fleischrindern; Tagungsband „Qualität von Futtermitteln und tierischen Primärprodukten“; Halle / Saale, 17. und 18. November 2000

STIEWE, H.:

In der Fütterung stecken die finaziellen Reserven; in top agrar extra: Fleischrinderproduktion, Landwirtschaftsverlag GmbH Münster-Hiltrup (1989), 40-45

STIEWE, H.:

Fütterung der Kälber; in top agrar extra: Fleischrinderproduktion; Landwirtschaftsverlag GmbH Münster-Hiltrup (1994), 47

STROH, T.L.; RINGWALL, K.A.; NELSON, J.L.; HELMUTH, K.J.; LANDBLOM, D.L.:

Evaluation of digestible fiber based creep feed diets and subsequent 28-day complete pelleted starting feed performance; Dickinson Research Extension Center - Report 2001, North Dakota State Univ. (2001)

TARR, S.L.; FAULKNER, D.B.; BUSKIRK, D.D.; IRELAND, F.A.; PARRETT, D.F.; BERGER, L.L.:

The value of creep feeding during the last 84, 56, or 28 days prior to weaning on growth performance of bursing calves grazing endophyte-infected tall fescue; J. Anim. Sci. 72 (1994), 1084-1094

TEICHMANN, S.; FAHR, R.-D.; LENGERKEN, G v..; MÖRCHEN, F.:

Futteraufnahme und Futterverwertung von Mutterkühen der Rassen Deutsch Angus und Fleckvieh in der frühen Laktation; Vortragstagung der DGfZ und der GfT (1998), D02 


\section{TEICHMANN, S.:}

Beziehungen zwischen Futteraufnahme, Milchmenge und Milchinhaltsstoffen, Energieretention sowie klinisch-chemischen Blutparametern bei Mutterkühen; Univ. Leipzig, Diss., 2001

TJARDES, K.E.; FAULKNER, D.B.; BUSKIRK, D.D.; PARRETT, D.F.; BERGER, L.L.; MERCHEN, N.R.;

IRELAND, F.A.:

The influence of processed corn and supplemental fat on digestion of limited-fed diets and performance of beef cows; J. Anim. Sci. 76 (1998), 8-17

WAGNER, D.G.; GILL, D.; LUSBY, K.S.:

Feeding cattle on grass; Oklahoma State Univ., Extension Facts No. 3011 (1998)

WARZECHA, H.:

Aktuelle Probleme in der Jungrinderaufzucht bei Weidenutzung in Thüringen; 2. Thüringer Nutztierforum „Kälber- und Jungrinderaufzucht“ Heft 8; Thüringer Landesanstalt für Landwirtschaft (1999)

WEIß, J.; PABST, W.; STRACK, K.E.; GRANZ, S.:

Tierproduktion; PAREY Verlag Berlin; 12., neubearbeitete Auflage 1998

WYATT, R.D.; GOULD, M.B.; WHITEMAN, J.V.; TOTUSEK, R.:

Effect of milk level and biological type on calf growth and performance; J. Anim. Sci. 45 (1977), 11381145

Eingegangen: 28.08.2002

Akzeptiert: 23.09.2002

Anschriften der Verfasser

Dipl.-Ing. (FH) HEIKO SCHOLZ, Dr. STEPHAN SCHÄFER,

Prof. Dr. habil. ROLF-DIETER FAHR

Institut für Tierzucht und Tierhaltung mit Tierklinik

Martin-Luther-Universität Halle-Wittenberg

Adam-Kuckhoff-Straße 35

D - 06108 Halle / Saale

\section{Dr. FRIEDRICH MÖRCHEN}

Lehr- und Versuchsanstalt für Tierhaltung und Technik Iden

Lindenstraße 18

D-39606 Iden 